\title{
The Use of Receiver Operating Characteristic (Roc) Analysis in the Evaluation of the Performance of Two Binary Diagnostic Tests of Gestational Diabetes Mellitus
}

\section{Okeh $\mathrm{UM}^{1 *}$ and Okoro $\mathrm{CN}^{2}$}

${ }^{1}$ Department of Industrial mathematics and Applied Statistics, Ebonyi State University, Abakaliki Nigeria

${ }^{2}$ Depatment of Industrial Mathematics and Applied Statistics, Ebonyi State University, Nigeria

\begin{abstract}
Objective: To compare the accuracy measures of the random glucose test and the $50-\mathrm{g}$ glucose challenge test as screening tests for gestational diabetes mellitus (GDM).

Research Design and Methods: In this prospective cohort study, pregnant women without preexisting diabetes in two perinatal centers in the Ebonyi State underwent a random glucose test and a 50-g glucose challenge test between 24 and 28 weeks of gestation. If one of the screening tests exceeded predefined threshold values, the $75-g$ oral glucose tolerance test (OGTT) was performed within 1 week. Furthermore, the OGTT was performed in a random sample of women in whom both screening tests were normal. GDM was considered present when the OGTT (reference test) exceeded predefined threshold values. Receiver operating characteristic (ROC) analysis was used to evaluate the performance of the two screening tests. The results were corrected for verification bias.

Results: We included 1,301 women. The OGTT was performed in 322 women. After correction for verification bias, the random glucose test showed an area under the ROC curve of $0.69(95 \% \mathrm{Cl} 0.61-0.78)$, whereas the glucose challenge test had an area under the curve of $0.88(0.83-0.93)$. There was a significant difference in area under the curve of the two tests of $0.19(0.11-0.27)$ in favor of the $50-g$ glucose challenge test.
\end{abstract}

Conclusions: In screening for GDM, the 50 -g glucose challenge test is more useful than the random glucose test.

Keywords: Random glucose test; 50 -g glucose challenge test; $75-\mathrm{g}$ oral glucose tolerance test; ROC curve

\section{Introduction}

Gestational diabetes mellitus (GDM) is estimated to occur in 2-9\% of all pregnancies [1-5]. It is defined as carbohydrate intolerance with onset or first recognition during pregnancy and is associated with increased rates of adverse pregnancy outcomes, such as macrosomia; shoulder dystocia; birth-related trauma, such as fractures and nerve palsies; neonatal hypoglycemia; and jaundice. In addition, women with GDM are at substantially higher risk to develop diabetes in later life $[1,6,7,8]$. Results from a randomized controlled trial show that treatment of GDM by means of dietary advice, blood glucose monitoring, and insulin therapy, if required, reduces the rate of serious perinatal complications without increasing the rate of caesarean delivery [1]. Based on these results, identification through screening and subsequent treatment of women with GDM appears beneficial. However, consensus on the optimal policy for screening is lacking. The American Diabetes Association [9] recommends screening based on risk factors for GDM (age > 25 years, obese, close relative with diabetes, history of GDM or a previous macrosomic infant, or specific ethnicity) followed by the 50-g 1-h oral glucose challenge test as a screening test $[10,11]$. Other methods of screening that are regularly used are (repeated) random glucose testing and fasting glucose measurement. It is indefinite which test is the most accurate in testing women for GDM. The diversity in screening methods may result in unidentified cases of GDM and preventable neonatal and maternal morbidity. Establishment of an optimal, evidence-based screening policy to detect and treat GDM in a timely fashion could contribute to a reduction of perinatal complications. Two regularly used screening tests in the Ebonyi State health system are the random glucose test and the 50-g glucose challenge test. The objective of the present study was to compare these two tests as screening tests for GDM as a first step in determining optimal screening policy in GDM.

\section{Receiver Operating Characteristic Curve}

ROC (Receiver Operating Characteristic) analysis is being used as a method for evaluation and comparison of classifiers [12]. The ROC gives complete description of classification accuracy as given by the area under the ROC curve. The ROC curve originates from signal detection theory [13]; the curve shows how the receiver operates the existence of signal in the presence of noise. The ROC curve plots the probability of detecting true signal (sensitivity) and false signal ( 1 - specificity) for an entire range of possible cut points. The sensitivity and specificity of a classifier also depend on the definition of the cut-off point for the probability of predicted classes. A ROC curve demonstrates the trade-off between true positive rate and false positive rate in binary classification problems. To draw a ROC curve, the true positive rate (TPR) and the false positive rate (FPR) are needed. TPR determines the performance of a classifier or a diagnostic test in classifying positive cases correctly among all positive samples available during the test.

*Corresponding author: Okeh UM, Department of Industrial mathematics and Applied Statistics, Ebonyi State University, Abakaliki, Nigeria, E-mail: umokeh1@yahoo.com

Received December 01, 2011; Accepted January 13, 2012; Published January 19, 2012

Citation: Okeh UM, Okoro CN (2012) The Use of Receiver Operating Characteristic (Roc) Analysis in the Evaluation of the Performance of Two Binary Diagnostic Tests of Gestational Diabetes Mellitus. J Biomet Biostat S7:002. doi:10.4172/21556180.S7-002

Copyright: (C) 2012 Okeh UM, et al. This is an open-access article distributed unde the terms of the Creative Commons Attribution License, which permits unrestricted use, distribution, and reproduction in any medium, provided the original author and source are credited. 
FPR, on the other hand, defines how many incorrect positive results, which are actually negative, there are among all negative samples available during the test. Because TPR is equivalent to sensitivity and FPR is equal to (1 -specificity), the ROC graph is sometimes called the sensitivity vs. ( 1 - specificity) plot. The area under the ROC curve has become a particularly important measure for evaluating classifiers' performance because it is the average sensitivity over all possible specificities [14]. The larger the area, the better the classifier performs. If the area is 1.0, the classifier achieves both $100 \%$ sensitivity and $100 \%$ specificity. If the area is 0.5 , then we have $50 \%$ sensitivity and $50 \%$ specificity, which is no better than flipping a coin. This single criterion can be compared for measuring the performance of different classifiers analyzing a dataset. $[15,16]$. After a classifier has been made, it is also useful to measure its calibration. Calibration evaluates the degree of correspondence between the estimated probabilities of a specific outcome resulting from a classifier and the outcomes predicted by domain experts. This can then be tested using goodness-of-fit statistics. This test examines the difference between the observed frequency and the expected frequency for groups of patients and can be used to determine if the classifier provides a good fit for the data. If the p-value is large, then the classifier is well calibrated and fits the data well. If the p-value is small, then the classifier is not well calibrated. There is a pair of diagnostic sensitivity and specificity values for every individual cut-off. To construct a ROC graph, we plot these pairs of values on the graph with the 1-specificity on the $\mathrm{x}$-axis and sensitivity on the $\mathrm{y}$-axis. Receiver operating characteristic curve analysis is often used to help determine the cut-off point to optimize sensitivity and specificity. An ROC curve is a graphical representation of the trade off between the false negative and false positive rates for every possible cut-off value [17]. Alternatively, the ROC curve is the representation of the trade off between sensitivity and specificity. In other words, the ability of a test using a specific analytic concentration, to discriminate disease from non-disease can be graphically portrayed by use of ROC curve analysis. A graph can be generated in which the sensitivity and specificity are determined for each data point obtained in the study. These are graphed with sensitivity of each data point on the y-axis and the corresponding 1 -specificity for each data point on the $\mathrm{x}$-axis. Precisely, we plot these pairs of values on the graph with the 1-specificity on the $\mathrm{x}$-axis and sensitivity on the $y$-axis. (Note: the ratio of the $y$-axis/x-axis is the likelihood ratio positive or the graph of true positives and false positives respectively). For the ideal test, the plot would rise from 0 and go straight up to 1.00 and then a horizontal line along the 1.00 sensitivity line. This would be where there is no overlap in the data points and sensitivity and specificity would both be $100 \%$ in the left hand corner[17]. This rarely occurs and more commonly a curvilinear plot is observed. The greater the area under the curve, the more discriminatory (the ability of the test to correctly classify those with and without the disease) the test is, ideally, the area under a curve of 1.00 is a perfectly discriminatory test and a curve that follows the diagonal line in the graph has an area under the curve 0.5 which corresponds to the test being no better than flipping a coin [17]. The shape of a ROC curve and the area under the curve (AUC) helps us estimate how high is the discriminative power of a test. The closer the curve is located to upperleft hand corner and the larger the area under the curve, the better the test is at discriminating between diseased and non-diseased. The area under the curve can have any value between 0 and 1 and it is a good indicator of the goodness of the test. A perfect diagnostic test has an AUC 1.0. whereas a nondiscrimination test has an area 0.5 . The larger the area under the curve, the better the diagnostic test in discriminating those with and without disease [17]. Many statistical programs can generate a table of the values in the graph and calculate sensitivity, specificity, LP+,LP-, and proportion or percent correctly identified for each data point. Cut-off points are not necessarily chosen to optimize the number of patients correctly categorized. One can select different cut-off points to optimize sensitivity or specificity. For example, when a screening test is used to look for a serious disease that if missed could result in serious harm to the patient, the sensitivity of that test should be optimized. Conversely, in situations where therapy could be extremely harmful if given to a patient without the disease, one would choose a cut-off point that optimizes specificity. In general, when optimizing one test characteristic, the other gets worse and vice versa. For example, when improving sensitivity, specificity decreases and when improving specificity, sensitivity decreases. The area under the ROC curve can also be used statistically to compare the discriminating ability between two diagnostic tests [17]. We can say that the relationship between the area under the ROC curve (AUC) and diagnostic accuracy can be seen in the table 1 below :

AUC is a global measure of diagnostic accuracy. It tells us nothing about individual parameters, such as sensitivity and specificity. Out of two tests with identical or similar AUC, one can have significantly higher sensitivity, whereas the other significantly higher specificity. Furthermore, data on AUC state nothing about predicative vales and about the contribution of the test in ruling-in and ruling-out a diagnosis. Global measures are there for general assessment and for comparison of two or more diagnostic tests. By the comparison of areas under the two ROC curves we can estimate which one of two tests is more suitable for distinguishing health from disease or any other two conditions of interest. It should be pointed out that this comparison should not be based on visual nor intuitive evaluation [18]. For this purpose we use statistic tests which evaluate the statistical significance of estimated difference between two AUC, with previously defined level of statistical significance $(\mathrm{P})$.

\section{Research Design and Methods}

In a prospective cohort study, all pregnant women attending the outpatient obstetric departments at the Ebonyi State University Teaching Hospital Abakaliki (EBSUTHAI) and Federal Medical Centre (FMC) Abakaliki during a 3-year study period from January 2007 to December 2009 were invited to participate. Women known to have preexisting diabetes were excluded from the study, as well as those who had not reported for prenatal care in one of two participating hospitals before 24 weeks of gestation. Only women who delivered after 28 weeks of gestation were included in the analysis.

\section{Data}

At intake, the following information was obtained: obstetric history, family history of diabetes, ethnicity (categorized as Igbo or non-Igbo), height, self reported weight (before pregnancy), age, and smoking habits (categorized as smoking or nonsmoking). BMI was calculated as weight in kilograms divided by the square of height in meters. The following data regarding pregnancy and outcome were recorded after

\begin{tabular}{|l|l|}
\hline Area & Diagnostic Accuracy \\
\hline $0.9-1.0$ & Excellent \\
\hline $0.8-0.9$ & Very good \\
\hline $0.7-0.8$ & Good \\
\hline $0.6-0.7$ & Sufficient \\
\hline $0.5-0.6$ & Bad \\
\hline$<0.5$ & Test not useful \\
\hline
\end{tabular}

Table 1: Relationship between the area under the ROC curve (AUC) and diagnostic accuracy. 
Citation: Okeh UM, Okoro CN (2012) The Use of Receiver Operating Characteristic (Roc) Analysis in the Evaluation of the Performance of Two Binary Diagnostic Tests of Gestational Diabetes Mellitus. J Biomet Biostat S7:002. doi:10.4172/2155-6180.S7-002

delivery: weight gain during pregnancy, treatment with diet or insulin, duration of pregnancy in days, birth weight of the neonate in grams, Apgar score after 1 and $5 \mathrm{~min}$, and arterial and venous $\mathrm{pH}$ from the umbilical cord. In all women, the random glucose test was performed at intake ( \pm 12 weeks) and between the 24th and 28th week of gestation. If the random plasma glucose measured between 24 and 28 weeks of gestation was $\geq 6.8 \mathrm{mmol} / \mathrm{l}$, the random glucose test was considered abnormal. If random plasma glucose measurement was not performed between the 24th and 28th week, a random plasma glucose at intake $\geq 6.8 \mathrm{mmol} / \mathrm{l}$ was considered indicative for GDM. A 50-g oral glucose challenge test was performed between the 24th and 28th week of gestation. The test was performed irrespective of time of the day and of the last meal. Plasma glucose was measured $1 \mathrm{~h}$ after administration of a solution containing $50 \mathrm{~g}$ of glucose. The predefined cutoff value for an abnormal test result was a 1-h plasma glucose value of $7.8 \mathrm{mmol} / \mathrm{l}$. If either the random glucose test or the $50 \mathrm{-g}$ oral glucose challenge test exceeded the predefined threshold value, a 2-h 75-g oral glucose tolerance test (OGTT) was performed within 1 week to confirm or rule out the presence of GDM (reference test). The OGTT was performed in the morning after a 12-h overnight fast and 3 days of minimal 150to 200 -g carbohydrate diet. Plasma glucose was determined before and $2 \mathrm{~h}$ after administration of a 75-g glucose-containing solution. GDM was considered present if venous plasma glucose equaled or exceeded the threshold values according to World Health Organization criteria ( $<7.8 \mathrm{mmol} / \mathrm{l}$ after 12 -h overnight fast and $\geq 7.8 \mathrm{mmol} / \mathrm{l}$ at $2 \mathrm{~h}$ after administration of a 75-g glucose-containing solution). These criteria were also applied in the randomized controlled trial in which treatment of GDM was considered beneficial [1].

\section{Verification bias}

When a screening test is evaluated against a reference test, ideally all participating patients should undergo both the screening and the reference test. However, in practice, the reference test is seldom performed in all patients, as this test is often more invasive or expensive. If only patients with verified screening test results are used to assess the performance of the screening test, calculated accuracy measures become biased because patients with verified disease status are often only patients with an abnormal screening test result, and, therefore, they do not represent a random sample of the population in which the screening test is used. The bias that occurs is called (partial) verification bias [19]. As in the present study, the reference test was, according to the predefined protocol, not performed in all patients. We used the following procedure to correct for verification bias. We performed the OGTT (reference test) in an arbitrary subset of consecutive patients with two negative screening test results to determine the extent to which cases of GDM were missed by the screening tests. Subsequently, we estimated OGTT measurements in women who were not subjected to an OGTT based on results of the random test and the 50-g glucose screening test as well as on patient characteristics using multiple logistic regression analysis. In other words, if the result of the OGTT was missing, OGTT values were estimated with multiple regression analysis, using the results of the two screening tests and available patient characteristics. This procedure to handle missing data is called imputation and is a commonly used adequate technique to correct for verification bias [20,21]. By using multiple imputation instead of single imputation (i.e., performing the imputation procedure multiple times instead of just once), uncertainty in the imputed values is reflected by the variation in imputed values across multiple imputed datasets and, thus, by appropriately larger SEs [22]. The multiple imputation procedure was also used to impute incidental missing data on patient characteristics.

\section{Statistical Analysis}

The distribution of continuous variables is reported as means \pm SD. We constructed two-by-two tables for abnormal and normal test results on the random glucose test and the 50 -g glucose screening test against the OGTT. These tables reflect true-positive, false-positive, true -negative, or false-negative test results for both the random glucose test and the 50-g glucose challenge test. Diagnostic accuracy (sensitivity, specificity, predictive values, and likelihood ratios) and 95\% CIs were calculated. Receiver operating characteristic (ROC) analysis was used to evaluate the discriminatory power of the two screening tests. Data were analyzed using SPSS 12.0.1 (SPSS, Chicago, IL) and SAS 9.1.3.

\section{Results of Analysis}

We included 1,305 women. Four women were excluded from analysis because they delivered before 28 weeks of gestation. Data from 1,301 women were used for further analysis. Patient characteristics are presented in Table 2 . Thereby, the distribution of patient characteristics within the classification groups of the reference test (OGTT) can be compared. Table 3 displays the position of patients in our study based on the results of the subsequent diagnostic tests. Of all 1,301 women, at least one test result of the random glucose test was obtained. The random glucose test was performed at intake and between the 24th and the $28^{\text {th }}$ week of gestation in $1,169(89.9 \%)$ and $1,295(99.5 \%)$

\begin{tabular}{|c|c|c|c|c|}
\hline & GDM present & $\begin{array}{l}\text { GDM not } \\
\text { present }\end{array}$ & $\begin{array}{l}\text { GDM not } \\
\text { verified }\end{array}$ & Total \\
\hline N & 46 & 276 & 979 & 1,301 \\
\hline Age (years) & $30.8 \pm 4.6$ & $30.6 \pm 4.9$ & $30.8 \pm 5.0$ & $30.8 \pm 4.9$ \\
\hline $\begin{array}{l}\text { BMI before pregnancy }(\mathrm{kg} / \\
\left.\mathrm{m}^{2}\right)\end{array}$ & $25.6 \pm 4.4$ & $25.7 \pm 5.2$ & $23.8 \pm 4.4$ & $24.2 \pm 4.6$ \\
\hline \multicolumn{5}{|l|}{ Ethnicity } \\
\hline Igbo & $37(82.2)$ & $247(90.5)$ & $848(89.4)$ & $1,132(89.4)$ \\
\hline Non-lgbo & $8(17.8)$ & $26(9.5)$ & $101(10.6)$ & $135(10.6)$ \\
\hline \multicolumn{5}{|l|}{ Family history of diabetes } \\
\hline Yes & $13(28.9)$ & $55(20.1)$ & 185 (19.5) & $253(19.7)$ \\
\hline No & $32(71.1)$ & 218 (79.9) & $783(80.5)$ & $1,033(80.3)$ \\
\hline \multicolumn{5}{|l|}{ Smoking } \\
\hline Yes & $8(17.4)$ & $46(17.0)$ & $170(17.5)$ & $224(17.4)$ \\
\hline No & $38(82.6)$ & $225(83.0)$ & $799(82.5)$ & $1,062(82.6)$ \\
\hline \multicolumn{5}{|l|}{ Hospital } \\
\hline EBSUTHAI & $22(47.8)$ & 99 (35.9) & $874(89.3)$ & 995 (76.5) \\
\hline FMC & $24(52.2)$ & $177(64.1)$ & $105(10.7)$ & $306(23.5)$ \\
\hline \multicolumn{5}{|l|}{ Obstetric history 1} \\
\hline $\begin{array}{l}\text { Previous spontaneous } \\
\text { abortion }\end{array}$ & $15(32.6)$ & $84(30.4)$ & $317(32.4)$ & $416(32.0)$ \\
\hline $\begin{array}{l}\text { Non-previous spontaneous } \\
\text { abortion }\end{array}$ & $31(67.4)$ & $192(69.6)$ & $662(67.6)$ & $885(68.0)$ \\
\hline \multicolumn{5}{|l|}{ Obstetric history 2} \\
\hline Nullipara & $19(43.2)$ & $112(40.9)$ & $422(44.3)$ & $553(43.5)$ \\
\hline $\begin{array}{l}\text { Multipara with history of } \\
\text { GDM }\end{array}$ & $2(4.5)$ & $8(2.9)$ & $6(0.6)$ & $16(1.3)$ \\
\hline $\begin{array}{l}\text { Multipara without history } \\
\text { of GDM }\end{array}$ & $23(52.3)$ & $154(56.2)$ & $525(55.1)$ & 702 (55.2) \\
\hline \multicolumn{5}{|l|}{ Obstetric history 3} \\
\hline Nullipara & $19(43.2)$ & $112(40.7)$ & $422(44.3)$ & $553(43.5)$ \\
\hline $\begin{array}{l}\text { Multipara with perinatal } \\
\text { mortality }\end{array}$ & $4(9.1)$ & $17(6.2)$ & $47(4.9)$ & $68(5.3)$ \\
\hline $\begin{array}{l}\text { Multipara without perinatal } \\
\text { mortality }\end{array}$ & $21(47.7)$ & $146(53.1)$ & $484(50.8)$ & $651(51.2)$ \\
\hline
\end{tabular}

Data are means \pm SD or $n(\%)$.

Table 2: Demographics before correction for verification bias. 
Citation: Okeh UM, Okoro CN (2012) The Use of Receiver Operating Characteristic (Roc) Analysis in the Evaluation of the Performance of Two Binary Diagnostic Tests of Gestational Diabetes Mellitus. J Biomet Biostat S7:002. doi:10.4172/2155-6180.S7-002

Page 4 of 6

\begin{tabular}{|c|c|c|c|c|c|c|c|c|c|c|c|c|c|c|}
\hline \multicolumn{15}{|c|}{1301 women met the inclusion criteria } \\
\hline \multicolumn{9}{|c|}{ Negative random glucose test- 1264} & \multicolumn{6}{|c|}{ Positive random glucose test-37 } \\
\hline \multicolumn{3}{|c|}{$\begin{array}{l}\text { Unknown } 50 \mathrm{~g} \text { glucose challenge } \\
\text { test-20 }\end{array}$} & \multicolumn{3}{|c|}{$\begin{array}{l}\text { Negative } 50 \mathrm{~g} \text { glucose challenge } \\
\text { test- } 1097\end{array}$} & \multicolumn{3}{|c|}{$\begin{array}{l}\text { Positive } 50 \mathrm{~g} \text { glucose challenge } \\
\text { test- } 147\end{array}$} & \multicolumn{3}{|c|}{$\begin{array}{l}\text { Negative } 50 \mathrm{~g} \text { glucose challenge } \\
\text { test- } 17\end{array}$} & \multicolumn{3}{|c|}{$\begin{array}{l}\text { Positve } 50 \mathrm{~g} \text { glucose challenge } \\
\text { test- } 20\end{array}$} \\
\hline $\begin{array}{l}\text { Negative } \\
\text { OGTT- } \\
3(19)\end{array}$ & $\begin{array}{l}\text { Positive } \\
\text { OGTT- } \\
1(1)\end{array}$ & $\begin{array}{l}\text { Unverified } \\
\text { OGTT- } \\
16(0)\end{array}$ & $\begin{array}{l}\text { Negative } \\
\text { OGTT- } \\
159(1084)\end{array}$ & $\begin{array}{l}\text { Positive } \\
\text { OGTT- } \\
13(13)\end{array}$ & $\begin{array}{l}\text { Unverified } \\
\text { OGTT- } \\
925(0)\end{array}$ & $\begin{array}{l}\text { Negative } \\
\text { OGTT- } \\
97(120)\end{array}$ & $\begin{array}{l}\text { Positive } \\
\text { OGTT- } \\
27(27)\end{array}$ & $\begin{array}{l}\text { Unverified } \\
\text { OGTT- } \\
23(0)\end{array}$ & $\begin{array}{l}\text { Negative } \\
\text { OGTT- } \\
8(16)\end{array}$ & $\begin{array}{l}\text { Positive } \\
\text { OGTT- } \\
1(1)\end{array}$ & $\begin{array}{l}\text { Unverified } \\
\text { OGTT- } \\
8(0)\end{array}$ & $\begin{array}{l}\text { Negative } \\
\text { OGTT- } \\
9(14)\end{array}$ & $\begin{array}{l}\text { Positive } \\
\text { OGTT- } \\
4(6)\end{array}$ & $\begin{array}{l}\text { Unverified } \\
\text { OGTT- } \\
7(0)\end{array}$ \\
\hline
\end{tabular}

Table 3: Screening and diagnostic test results before and after correction for verification bias. The figures in the table represent the number of women with the specific combination of test results before (after) correction of verification bias. Figures between parentheses represent the number of women after correction for verification bias.

of the 1,301 women, respectively. We used the results of the random glucose test obtained at intake for the six women $(0.5 \%)$ in whom the random glucose measurement was not performed between the 24 th and the 28 th week of gestation. None of these six women had a random glucose test result $>6.8 \mathrm{mmol} / \mathrm{l}$. The 50 -g oral glucose challenge test was performed in 1,281 women (98.5\%). There were 37 of 1,301 women $(2.8 \%)$ who had an abnormal random glucose test, whereas 167 of 1,281 women (13.0\%) had an abnormal 50 -g glucose challenge test. There were 184 women (14.1\%) with at least one abnormal test result (random glucose test or 50-g glucose challenge test or both). In 20 women (1.5\%), both tests results were suspect for GDM. The OGTT was performed in 322 women (24.8\%). This included 146 of 184 women $(79.3 \%)$ with an abnormal screening test result and a subgroup of 176 women with two negative screening tests (Table 3). Initially, GDM was diagnosed in 46 women. After correction for verification bias, 48 women were diagnosed with GDM (3.7\%). We used multiple imputation of the OGTT value for every patient in whom the OGTT was not performed. This would have been an adequate procedure if the chance of verification of a screening test result depended solely on the result of the screening test. However, we calculated that the chance of verification was not completely independent of factors other than the results of the screening tests. In general, women with a history of GDM or perinatal death, increased BMI, and women from the Federal Medical Centre (FMC) hospital in Abakaliki were more likely to be verified, independent of the results of their screening tests. Due to this nonrandom verification, there was a high prevalence of GDM in women with two negative screening tests who underwent an OGTT. As a result, the prevalence of GDM in the imputed dataset became unrealistically high (up to $15 \%$ ). To obtain imputed data that are in line with the incidence of GDM in the Ebonyi State (estimated to be 2-4\%), we adjusted the imputation procedure by applying the following additional criterion to limit the number of cases classified as having GDM. Based on the same covariates (screening tests and patient characteristics), multiple imputation was repeated 100 times and unverified women were only classified as having GDM if they had consistently imputed OGTT values that were indicative for GDM $(>75 \%)$. After this adjusted multiple imputation procedure, the prevalence of GDM in our sample was $3.7 \%$. Only two unverified women were classified as having GDM, whereas in all other women that were unverified no GDM was assumed. Table 2 displays results of the comparison of the two screening tests in terms of accuracy measures calculated after correction for verification bias. Comparison of accuracy measures after correction for verification bias resulted in an almost five times- higher sensitivity in favor of the 50 -g glucose challenge test compared with the random glucose test (70.2\% [95\% CI 57.1- 83.3] vs. $14.6 \%$ [ $4.6-24.6])$. The random glucose test had less false-positive test results and was therefore more specific (97.6\% [96.6 -98.5] vs. 89.1\% [87.4 -90.9]). Positive predictive values for both tests were comparable, as were the negative predictive values. The likelihood ratio of an abnormal test result was larger for the 50-g glucose challenge test than for the random glucose test. The likelihood ratio of a normal test was smaller for the 50 -g glucose challenge test.
The area under the ROC curve was larger for the 50-g glucose challenge test $(0.88[0.83-0.93])$ than for the random glucose test $(0.69[0.61-$ $0.78]$ ). There was a significant difference in the areas under the curve of the two tests of $0.19(0.11-0.27)$. See Figure for the area under the ROC curve showing that GCT is a better test when compared to random glucose test (Figure 2).

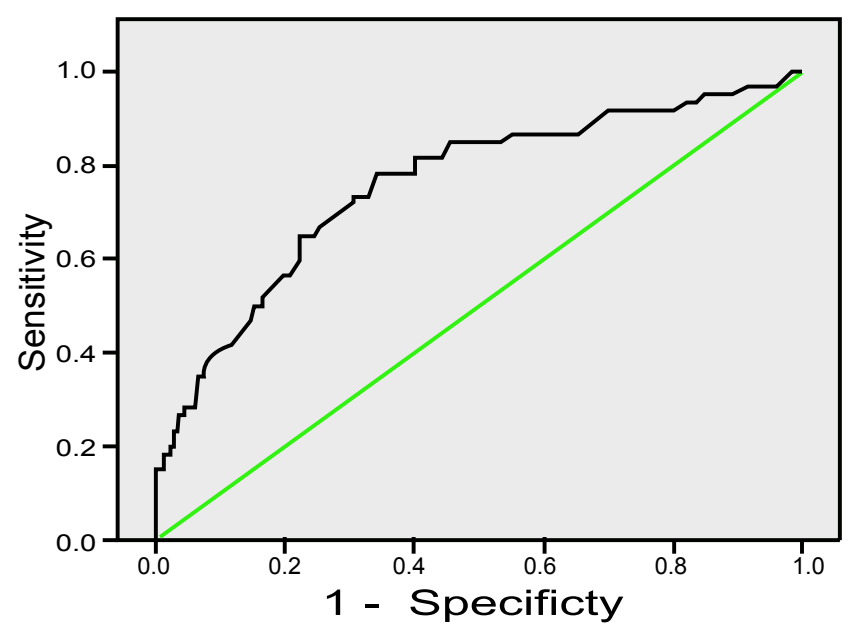

Figure 1: $50 \mathrm{~g}$ Glucose Challenge Test (GCT) with AUC=0.88(95\% C.I=083-0.93).

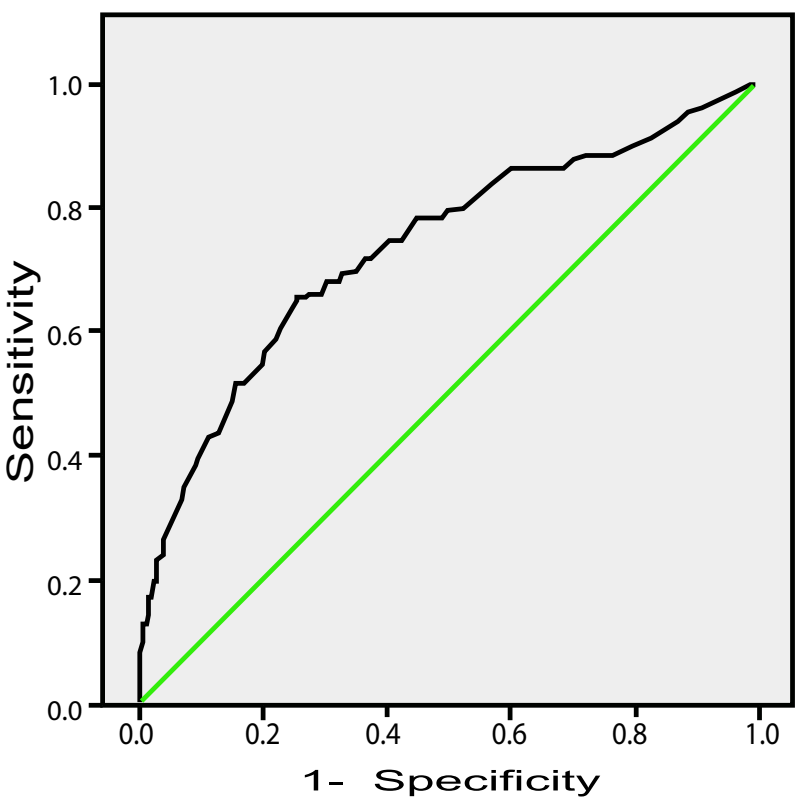

Figure 2: Random Glucose test with AUC=0.69(95\% C.I=0.61-0.78). 
Citation: Okeh UM, Okoro CN (2012) The Use of Receiver Operating Characteristic (Roc) Analysis in the Evaluation of the Performance of Two Binary Diagnostic Tests of Gestational Diabetes Mellitus. J Biomet Biostat S7:002. doi:10.4172/2155-6180.S7-002

Page 5 of 6

\begin{tabular}{|c|c|c|c|c|c|c|c|c|}
\hline & \multicolumn{4}{|c|}{ Random glucose test } & \multicolumn{4}{|c|}{ 1-h $50-g$ glucose challenge test } \\
\hline & OGTT Positive & OGTT Negative & OGTT unverified & Total & OGTT Negative & OGTT positive & OGTT unverified & Total \\
\hline $\begin{array}{l}\text { Positive Screening } \\
\text { test }\end{array}$ & 7 & 30 & 0 & 37 & 33 & 134 & 0 & 167 \\
\hline $\begin{array}{l}\text { Negative Screening } \\
\text { test }\end{array}$ & 41 & 1223 & 0 & 1264 & 14 & 1100 & 0 & 1114 \\
\hline Total & 48 & 1253 & 0 & 1301 & 47 & 1234 & 0 & 1281 \\
\hline Sensitivity (\%) & \multicolumn{4}{|l|}{$14.6(4.6-24.6)$} & \multicolumn{4}{|l|}{$70.2(57.1-83.3)$} \\
\hline Specificity (\%) & \multicolumn{4}{|l|}{$97.6(96.6-98.5)$} & \multicolumn{4}{|l|}{$89.1(87.4-90.9)$} \\
\hline PPV $(\%)$ & \multicolumn{4}{|l|}{$18.9(6.3-31.5)$} & \multicolumn{4}{|l|}{$19.8(3.7-25.8)$} \\
\hline NPV $(\%)$ & \multicolumn{4}{|l|}{$96.8(91.0-100.0)$} & \multicolumn{4}{|l|}{$98.7(97.1-100.0)$} \\
\hline LR (+ test result) & \multicolumn{4}{|l|}{$6.1(2.8-13.2)$} & \multicolumn{4}{|l|}{$6.5(5.1-8.3)$} \\
\hline LR (- test result) & \multicolumn{4}{|l|}{$0.88(0.78-0.98)$} & \multicolumn{4}{|l|}{$0.33(0.22-0.52)$} \\
\hline DOR & \multirow{2}{*}{\multicolumn{4}{|c|}{$\begin{array}{l}7.0(2.9-16.8) \\
0.69(0.61-0.78)\end{array}$}} & \multicolumn{4}{|l|}{$19.4(6.8-31.9)$} \\
\hline Area under the curve & & & & & \multicolumn{4}{|l|}{$0.88(0.83-0.93)$} \\
\hline
\end{tabular}

Table 4: Results of the $2 \times 2$ table and accuracy measures calculated after correction for verification bias.

\section{Conclusions}

Evidence for screening for GDM is often inconsistent and difficult to interpret due to various screening methods and thresholds applied internationally. An evidence-based policy could increase the number of identified women with GDM and therefore reduce the number of neonatal and maternal complications by providing adequate monitoring and treatment for these women. For this purpose, the present study compared the random glucose test and the 50-g glucose challenge test as screening tests for GDM. The area under the curve was larger for the $50 \mathrm{-g}$ glucose challenge test, indicating that the $50 \mathrm{-g}$ glucose challenge test was a better predictor for GDM than the random glucose test. A potential weakness in the present study is the number of missing reference tests, due to which verification bias occurred. Because verification was apparently not performed at random, characteristics other than the screening test results influenced the chance of verification. An intuitive and straightforward procedure to correct for verification bias would be to calculate the ratio of diseased to nondiseased from the results of the verified patients stratified by screening test results and to extrapolate this ratio to the unverified patients $[19,23]$. However, this mathematical correction can only be applied if verification of patients is performed completely at random or, in other words, if the chance of verification is truly independent of other factors such as, for example, patient characteristics. In addition, this results in an adjustment at the sample level. As for individual unverified patients, the disease status according to the reference test remains unknown (Table 4).

All accuracy measures are displayed with 95\% CIs estimate disease status accounting for these factors [24]. There are several strategies to deal with incomplete data, also within the context of partial verification [24]. As in our study, various imputation strategies consistently lead to a considerable higher number of cases, consequently implying unrealistically high prevalence rates. We therefore had to apply an additional criterion to limit the number of cases classified as having GDM by means of repeating the multiple imputation procedure for the OGTT 100 times and only classifying women as having GDM if they had consistently imputed values for the OGTT that were indicative for GDM ( $>75$ of 100 times). Further research is required to evaluate which approach is preferred, thereby also accounting for the epidemiological context of the study. The overall prevalence of GDM in the literature varies from 2 to $9 \%$ [1]. In the eastern part of Nigeria where Ebonyi State is found, the incidence is more often toward $2 \%$ than $9 \%$. Hypothetically, the incidence of GDM could be systematically underestimated in the literature (if these estimates have been based solely on selectively verified patients). In that case, we also underestimated the incidence of GDM and consequently our approach would have been suboptimal.
However, it is not very plausible that for years the incidence of GDM has been underestimated, so application of the described method should have corrected properly for this verification bias $[25,26]$. Results from the present study show that the 50 -g glucose challenge test has an almost fivefold higher sensitivity compared with random glucose testing. To our knowledge, these two screening tests have only been equated in the same sample two times before. McElduff et al. [27] found their results in favor of the 50-g challenge test, whereas Mathai et al. [28] found similar sensitivity for both tests and a higher specificity for the random test if both tests were performed in the 26th to 30th week of gestation. A number of studies compared the $50 \mathrm{-g}$ glucose challenge test with measurement of fasting glucose. Perucchini et al. [29] found the results in favor of the fasting glucose measurement, whereas Rey et al. [30] showed the 50-g glucose challenge test to be superior. Other studies investigating the test characteristics of the glucose challenge test reported sensitivities ranging from 58 to $80 \%$ $[31,32]$ for a specificity of $\sim 65 \%$ [32]. In these studies, thresholds for an abnormal result of the challenge test ranged from 7.2 to $7.8 \mathrm{mmol} / \mathrm{l}$. In the present study, a predefined cutoff value for an abnormal test result was set at $7.8 \mathrm{mmol} / \mathrm{l}$. If thresholds were set $<7.8 \mathrm{mmol} / \mathrm{l}$, sensitivity of the 50-g glucose challenge test would increase at the expense of a decreased specificity. The random glucose test is a fast, simple, and relatively inexpensive test. Accuracy of random glucose measurement is less frequently studied than that of the glucose challenge test. Nasrat et al. [33] evaluated random glucose measurement, which revealed a sensitivity of $16 \%$ and a specificity of $96 \%$ using a threshold value of 7.0 $\mathrm{mmol} / \mathrm{l}$ or $6.4 \mathrm{mmol} / \mathrm{l}$ if evaluated $\geq 2 \mathrm{~h}$ postprandial. Jowett et al. [34] also concluded that random glucose measurement is not sufficiently sensitive for screening on GDM. Results from the present study are in accordance with results from those two groups, using a threshold value for an abnormal test result of $6.8 \mathrm{mmol} / \mathrm{l}$. As high sensitivity is key to any screening test, random glucose testing is not an accurate method to screen women for GDM because five of six women with GDM would still be missed. In conclusion, we recommend that despite easy implementation, low costs, and relative high specificity, random glucose measurement should not be used as a screening test for GDM. Until superior screening alternatives become available, the 50-g glucose challenge test should be the preferred screening test for GDM.

\section{References}

1. Crowther CA, Hiller JE, Moss JR, McPhee AJ, Jeffries WS, et al. (2005) Effect of treatment of gestational diabetes mellitus on pregnancy outcomes. N Engl J Med 352: 2477-2486.

2. Esakoff TF, Cheng YW, Caughey AB (2005) Screening for gestational diabetes: different cut-offs for different ethnicities? Am J Obstet Gynecol 193: 1040-1044 
Citation: Okeh UM, Okoro CN (2012) The Use of Receiver Operating Characteristic (Roc) Analysis in the Evaluation of the Performance of Two Binary Diagnostic Tests of Gestational Diabetes Mellitus. J Biomet Biostat S7:002. doi:10.4172/2155-6180.S7-002

Page 6 of 6

3. Jensen DM, Damm P, Sorensen B, Molsted- Pedersen L, Westergaard JG, et al. (2003) Proposed diagnostic thresholds for gestational diabetes mellitus according to a $75-\mathrm{g}$ oral glucose tolerance test: maternal and perinatal outcomes in 3260 Danish women. Diabet Med 20: 51-57.

4. Sivan E, Weisz B, Homko CJ, Reece EA, Schiff E (2001) One or two hours postprandial glucose measurements: are they the same? Am J Obstet Gynecol 185: 604-607.

5. Tuffnell DJ, West J, Walkinshaw SA (2003) Treatments for gestational diabetes and impaired glucose tolerance in pregnancy. Cochrane Database Syst Rev CD003395.

6. Brody SC, Harris R, Lohr K (2003) Screening for gestational diabetes: a summary of the evidence for the U.S. Preventive Services Task Force. Oyster Gynecol 101: 380-392.

7. Langer O, Yogev Y, Most O, Xenakis EM (2005) Gestational diabetes: the consequences of not treating. Am J Obstet Gynecol 192: 989-997.

8. Naylor CD, Sermer M, Chen E, Farine D (1997) Selective screening for gestational diabetes mellitus: Toronto Trihospital Gestational Diabetes Project Investigators. N Engl J Med 337: 1591-1596.

9. American Diabetes Association (2003) Gestational diabetes mellitus. Diabetes Care 26: S103-S105

10. Poyhonen-Alho MK, Teramo KA, Kaaja RJ, Hiilesmaa VK (2005) 50 gram oral glucose challenge test combined with risk factor based screening for gestational diabetes. Eur J Obstet Gynecol Reprod Biol 121: 34-37.

11. Santos-Ayarzagoitia M, Salinas-Martinez AM, Villarreal-Perez JZ (2006) Gestational diabetes: validity of ADA and WHO diagnostic criteria using NDDG as the reference test. Diabetes Res Clin Pract 74: 322-328.

12. Ferri C, Flach P, Hernandez-Orallo J (2002) Learning Decision Trees Using the Area under the ROC Curve. Nineteenth International Conference on Machine Learning (ICML 2002), Morgan Kaufmann, 46-139.

13. Hosmer DW, Lemeshow S (2000) Applied Logistic Regression, Second Edition, Wiley, Inc., New York.

14. Bradley AP (1997) The use of the area under the roc curve in the evaluation of machine learning algorithms. Pattern Recognition 30: 1145-1159.

15. Hanley JA, McNeil BJ (1982) The meaning and use of the area under a receiver operating characteristic (ROC) curve. Radiology 143: 29-36.

16. Bamber D (1975) The area above the ordinal dominance graph and the area below the receiver operating characteristic graph. J Math Psychol 12: 387-415.

17. Zweig M, Campbell G (1993) Receiver operating characteristic (ROC) plots: a fundamental evaluation tool in clinical medicine. Clin Chem 39: 561-577.

18. Obuchowski NA, Lieber ML, Wians FH Jr (2004) ROC curves in clinical chemistry: uses, misuses, and possible solutions. Clin Chem 50: 1118-1125.
19. Begg CB (1987) Biases in the assessment of diagnostic tests. Stat Med 6 : 411-423.

20. Rubin DB (1987) Multiple Imputation for Nonresponse in Surveys. New York, John Wiley and Sons.

21. Schafer JL (1997) Analysis of Incomplete Multivariate Data. New York Chapman and Hall.

22. Frank Harrell (2001) Regression Modeling Strategies. New York, Springer

23. Begg CB, Greenes RA (1983) Assessment of diagnostic tests when disease verification is subject to selection bias. Biometrics 39: 207-215.

24. Pepe MS (2003) The Statistical Evaluation of Medical Tests for Classification and Prediction. Oxford, UK, Oxford University Press.

25. Hunink MG, Richardson DK, Doubilet PM, Begg CB (1990) Testing for fetal pulmonary maturity: ROC analysis involving covariates, verification bias, and combination testing. Med Decis Making 10: 201-211.

26. Pepe MS, Alonzo TA (2001) Comparing disease screening tests when true disease status is ascertained only for screen positives. Biostatistics 2: 249-260.

27. McElduff A, Goldring J, Gordon P, Wyndham L (1994) A direct comparison of the measurement of a random plasma glucose and a post-50 g glucose load glucose, in the detection of gestational diabetes. Aust N Z J Obstet Gynaecol 34: $28-30$

28. Mathai M, Thomas TJ, Kuruvila S, Jairaj P (1994) Random plasma glucose and the glucose challenge test in pregnancy. Natl Med J India 7:160-162.

29. Perucchini D, Fischer U, Spinas GA, Huch R, Hush A, et al. (1999) Using fasting plasma glucose concentrations to screen for gestational diabetes mellitus: prospective population based study. BMJ 319: 812-815.

30. Rey E, Hudon L, Michon N, Boucher P, Ethier J, et al. (2004) Fasting plasma glucose versus glucose challenge test: screening for gestational diabetes and cost effectiveness. Clin Biochem 37: 780-784.

31. de Sereday MS, Damiano MM, Gonzalez CD, Bennett PH (2003) Diagnostic criteria for gestational diabetes in relation to pregnancy outcome. J Diabetes Complications 17: 115-119

32. Maresh M (2005) Screening for gestational diabetes mellitus. Semin Feta Neonatal Med 10: 317-323.

33. Nasrat AA, Johnstone FD, Hasan SA (1988) Is random plasma glucose an efficient screening test for abnormal glucose tolerance in pregnancy? $\mathrm{Br}$ Obstet Gynaecol 95: 855- 860 .

34. Jowett NI, Samanta AK, Burden AC (1987) Screening for diabetes in pregnancy: is a random blood glucose enough?. Diabet Med 4: 160-163.
This article was originally published in a special issue, Medical statistics: Clinical and experimental research handled by Editor(s). Dr. Herbert Pang, Duke University, USA. 